\title{
A Century of Controversy over the Foundations of Mathematics
}

\author{
G. J. Chaitin, chaitin@watson.ibm.com
}

Lecture given Friday 30 April 1999 at UMass-Lowell. The lecture was videotaped; this is an edited transcript.

\section{Prof. Ray Gumb}

We're happy to have Gregory Chaitin from IBM's Thomas J. Watson Research Lab to speak with us today. He's a world-renowned figure, and the developer as a teenager of the theory of algorithmic information. And his newest book which is accessible to undergraduates, and I hope will be of great appeal to our undergraduates in particular, is available on the Web and comes with LISP programs to run with it. It's kind of like a combination of mathematics, computer science, and philosophy. Greg- 


\section{Greg Chaitin}

Thanks a lot! Okay, a great pleasure to be here! [Applause] Thank you very much! I'm awfully sorry to be late! You've got a beautiful town here! Those old brick buildings and the canals are really breathtaking! And thanks for being here for this talk! It's such a beautiful spring day - I think one has to be crazy to be indoors!

Okay, I'd like to talk about some crazy stuff. The general idea is that sometimes ideas are very powerful. I'd like to talk about theory, about the computer as a concept, a philosophical concept.

We all know that the computer is a very practical thing out there in the real world! It pays for a lot of our salaries, right? But what people don't remember as much is that really - I'm going to exaggerate, but I'll say it - the computer was invented in order to help to clarify a question about the foundations of mathematics, a philosophical question about the foundations of mathematics.

Now that sounds absurd, but there's some truth in it. There are actually lots of threads that led to the computer, to computer technology, which come from mathematical logic and from philosophical questions about the limits and the power of mathematics.

The computer pioneer Turing was inspired by these questions. Turing was trying to settle a question of Hilbert's having to do with the philosophy of mathematics, when he invented a thing called the Turing machine, which is a mathematical model of a toy computer. Turing did this before there were any real computers, and then he went on to actually build computers. The first computers in England were built by Turing.

And von Neumann, who was instrumental in encouraging the creation of computers as a technology in the United States, (unfortunately as part of the war effort, as part of the effort to build the atom bomb), he knew Turing's work very well. I learned of Turing by reading von Neumann talking about the importance of Turing's work.

So what I said about the origin of the computer isn't a complete lie, but it is a forgotten piece of intellectual history. In fact, let me start off with the final conclusion of this talk... In a way, a lot of this came from work of Hilbert. Hilbert, who was a very well-known German mathematician around the beginning of this century, had pro- 
posed formalizing completely all of mathematics, all of mathematical reasoning - deduction. And this proposal of his is a tremendous, glorious failure!

In a way, it's a spectacular failure. Because it turned out that you couldn't formalize mathematical reasoning. That's a famous result of Gödel's that I'll tell you about, done in 1931.

But in another way, Hilbert was really right, because formalism has been the biggest success of this century. Not for reasoning, not for deduction, but for programming, for calculating, for computing, that's where formalism has been a tremendous success. If you look at work by logicians at the beginning of this century, they were talking about formal languages for reasoning and deduction, for doing mathematics and symbolic logic, but they also invented some early versions of programming languages. And these are the formalisms that we all live with and work with now all the time! They're a tremendously important technology.

So formalism for reasoning did not work. Mathematicians don't reason in formal languages. But formalism for computing, programming languages, are, in a way, what was right in the formalistic vision that goes back to Hilbert at the beginning of this century, which was intended to clarify epistemological, philosophical questions about mathematics.

So I'm going to tell you this story, which has a very surprising outcome. I'm going to tell you this surprising piece of intellectual history.

\section{The Crisis in Set Theory}

So let me start roughly a hundred years ago, with Cantor...

\section{Georg Cantor}

The point is this. Normally you think that pure mathematics is static, unchanging, perfect, absolutely correct, absolute truth. . Right? Physics may be tentative, but math, things are certain there! Well, it turns out that's not exactly the case.

In this century, in this past century there was a lot of controversy over the foundations of mathematics, and how you should do math, 
and what's right and what isn't right, and what's a valid proof. Blood was almost shed over this... People had terrible fights and ended up in insane asylums over this. It was a fairly serious controversy. This isn't well known, but I think it's an interesting piece of intellectual history.

More people are aware of the controversy over relativity theory. Einstein was very controversial at first. And then of the controversy over quantum mechanics. . These were the two revolutions in the physics of this century. But what's less well known is that there were tremendous revolutions and controversies in pure mathematics too. I'd like to tell you about this. It really all starts in a way from Cantor.

\section{Georg Cantor}

What Cantor did was to invent a theory of infinite sets.

\section{Infinite Sets}

He did it about a hundred years ago; it's really a little more than a hundred years ago. And it was a tremendously revolutionary theory, it was extremely adventurous. Let me tell you why.

Cantor said, let's take $1,2,3, \ldots$

$$
1,2,3, \ldots
$$

We've all seen these numbers, right?! And he said, well, let's add an infinite number after this.

$$
1,2,3, \ldots \omega
$$

He called it $\omega$, lowercase Greek omega. And then he said, well, why stop here? Let's go on and keep extending the number series.

$$
1,2,3, \ldots \omega, \omega+1, \omega+2, \ldots
$$

Omega plus one, omega plus two, then you go on for an infinite amount of time. And what do you put afterwards? Well, two omega? (Actually, it's omega times two for technical reasons.)

$$
1,2,3, \ldots \omega \ldots 2 \omega
$$


Then two omega plus one, two omega plus two, two omega plus three, two omega plus four...

$$
1,2,3, \ldots 2 \omega, 2 \omega+1,2 \omega+2,2 \omega+3,2 \omega+4, \ldots
$$

Then you have what? Three omega, four omega, five omega, six omega, $\cdots$

$$
1,2,3, \ldots 3 \omega \ldots 4 \omega \ldots 5 \omega \ldots 6 \omega \ldots
$$

Well, what will come after all of these? Omega squared! Then you keep going, omega squared plus one, omega squared plus six omega plus eight... Okay, you keep going for a long time, and the next interesting thing after omega squared will be? Omega cubed! And then you have omega to the fourth, omega to the fifth, and much later?

$$
1,2,3, \ldots \omega \ldots \omega^{2} \ldots \omega^{3} \ldots \omega^{4} \ldots \omega^{5}
$$

Omega to the omega!

$$
1,2,3, \ldots \omega \ldots \omega^{2} \ldots \omega^{\omega}
$$

And then much later it's omega to the omega to the omega an infinite number of times!

$$
1,2,3, \ldots \omega \ldots \omega^{2} \ldots \omega^{\omega} \ldots \omega^{\omega^{\omega^{\omega \cdots}}}
$$

I think this is usually called epsilon nought.

$$
\varepsilon_{0}=\omega^{\omega^{\omega^{\omega \cdots}}}
$$

It's a pretty mind-boggling number! After this point things get a little complicated...

And this was just one little thing that Cantor did as a warm-up exercise for his main stuff, which was measuring the size of infinite sets! It was spectacularly imaginative, and the reactions were extreme. Some people loved what Cantor was doing, and some people thought that he should be put in an insane asylum! In fact he had a nervous breakdown as a result of those criticisms. Cantor's work was very influential, leading to point-set topology and other abstract fields in the mathematics of the twentieth century. But it was also very controversial. Some people said, it's theology, it's not real, it's a fantasy world, it has nothing to do with serious math! And Cantor never got a good position and he spent his entire life at a second-rate institution. 


\section{Bertrand Russell's Logical Paradoxes}

Then things got even worse, due mainly, I think, to Bertrand Russell, one of my childhood heroes.

\section{Bertrand Russell}

Bertrand Russell was a British philosopher who wrote beautiful essays, very individualistic essays, and I think he got the Nobel prize in literature for his wonderful essays. Bertrand Russell started off as a mathematician and then degenerated into a philosopher and finally into a humanist; he went downhill rapidly! [Laughter] Anyway, Bertrand Russell discovered a whole bunch of disturbing paradoxes, first in Cantor's theory, then in logic itself. He found cases where reasoning that seemed to be okay led to contradictions.

And I think that Bertrand Russell was tremendously influential in spreading the idea that there was a serious crisis and that these contradictions had to be resolved somehow. The paradoxes that Russell discovered attracted a great deal of attention, but strangely enough only one of them ended up with Russell's name on it! For example, one of these paradoxes is called the Burali-Forti paradox, because when Russell published it he stated in a footnote that it had been suggested to him by reading a paper by Burali-Forti. But if you look at the paper by Burali-Forti, you don't see the paradox!

But I think that the realization that something was seriously wrong, that something was rotten in the state of Denmark, that reasoning was bankrupt and something had to be done about it pronto, is due principally to Russell. Alejandro Garciadiego, a Mexican historian of math, has written a book which suggests that Bertrand Russell really played a much bigger role in this than is usually realized: Russell played a key role in formulating not only the Russell paradox, which bears his name, but also the Burali-Forti paradox and the Berry paradox, which don't. Russell was instrumental in discovering them and in realizing their significance. He told everyone that they were important, that they were not just childish word-play.

Anyway, the best known of these paradoxes is called the Russell paradox nowadays. You consider the set of all sets that are not members of themselves. And then you ask, "Is this set a member of itself or not?" 
If it is a member of itself, then it shouldn't be, and vice versa! It's like the barber in a small, remote town who shaves all the men in the town who don't shave themselves. That seems pretty reasonable, until you ask "Does the barber shave himself?" He shaves himself if and only if he doesn't shave himself, so he can't apply that rule to himself!

Now you may say, "Who cares about this barber!" It was a silly rule anyway, and there are always exceptions to the rule! But when you're dealing with a set, with a mathematical concept, it's not so easy to dismiss the problem. Then it's not so easy to shrug when reasoning that seems to be okay gets you into trouble!

By the way, the Russell paradox is a set-theoretic echo of an earlier paradox, one that was known to the ancient Greeks and is called the Epimenides paradox by some philosophers. That's the paradox of the liar: "This statement is false!" "What I'm now saying is false, it's a lie." Well, is it false? If it's false, if something is false, then it doesn't correspond with reality. So if I'm saying this statement is false, that means that it's not false - which means that it must be true. But if it's true, and I'm saying it's false, then it must be false! So whatever you do you're in trouble!

So you can't get a definite logical truth value, everything flip flops, it's neither true nor false. And you might dismiss this and say that these are just meaningless word games, that it's not serious. But Kurt Gödel later built his work on these paradoxes, and he had a very different opinion.

\section{Kurt Gödel}

He said that Bertrand Russell made the amazing discovery that our logical intuitions, our mathematical intuitions, are self-contradictory, they're inconsistent! So Gödel took Russell very seriously, he didn't think that it was all a big joke.

Now I'd like to move on and tell you about David Hilbert's rescue plan for dealing with the crisis provoked by Cantor's set theory and by Russell's paradoxes.

David Hilbert 


\section{David Hilbert to the Rescue with Formal Axiomatic Theories}

One of the reactions to the crisis provoked by Cantor's theory of infinite sets, one of the reactions was, well, let's escape into formalism. If we get into trouble with reasoning that seems okay, then one solution is to use symbolic logic, to create an artificial language where we're going to be very careful and say what the rules of the game are, and make sure that we don't get the contradictions. Right? Because here's a piece of reasoning that looks okay but it leads to a contradiction. Well, we'd like to get rid of that. But natural language is ambiguous - you never know what a pronoun refers to. So let's create an artificial language and make things very, very precise and make sure that we get rid of all the contradictions! So this was the notion of formalism.

\section{Formalism}

Now I don't think that Hilbert actually intended that mathematicians should work in such a perfect artificial language. It would sort of be like a programming language, but for reasoning, for doing mathematics, for deduction, not for computing, that was Hilbert's idea. But he never expressed it that way, because there were no programming languages back then.

So what are the ideas here? First of all, Hilbert stressed the importance of the axiomatic method.

\section{Axiomatic Method}

The notion of doing mathematics that way goes back to the ancient Greeks and particularly to Euclidean geometry, which is a beautifully clear mathematical system. But that's not enough; Hilbert was also saying that we should use symbolic logic.

Symbolic Logic

And symbolic logic also has a long history: Leibniz, Boole, Frege, Peano... These mathematicians wanted to make reasoning like algebra. Here's how Leibniz put it: He talked about avoiding disputes - and he 
was probably thinking of political disputes and religious disputes - by calculating who was right instead of arguing about it! Instead of fighting, you should be able to sit down at a table and say, "Gentleman, let us compute!" What a beautiful fantasy!...

So the idea was that mathematical logic should be like arithmetic and you should be able to just grind out a conclusion, no uncertainty, no questions of interpretation. By using an artificial math language with a symbolic logic you should be able to achieve perfect rigor. You've heard the word "rigor", as in "rigor mortis", used in mathematics? [Laughter] It's not that rigor! But the idea is that an argument is either completely correct or else it's total nonsense, with nothing in between. And a proof that is formulated in a formal axiomatic system should be absolutely clear, it should be completely sharp!

In other words, Hilbert's idea was that we should be completely precise about what the rules of the game are, and about the definitions, the elementary concepts, and the grammar and the language - all the rules of the game - so that we can all agree on how mathematics should be done. In practice it would be too much work to use such a formal axiomatic system, but it would be philosophically significant because it would settle once and for all the question of whether a piece of mathematical reasoning is correct or incorrect.

Okay? So Hilbert's idea seemed fairly straightforward. He was just following the axiomatic and the formal traditions in mathematics. Formal as in formalism, as in using formulas, as in calculating! He wanted to go all the way, to the very end, and formalize all of mathematics, but it seemed like a fairly reasonable plan. Hilbert wasn't a revolutionary, he was a conservative... The amazing thing, as I said before, was that it turned out that Hilbert's rescue plan could not work, that it couldn't be done, that it was impossible to make it work!

Hilbert was just following the whole mathematics tradition up to that point: the axiomatic method, symbolic logic, formalism...He wanted to avoid the paradoxes by being absolutely precise, by creating a completely formal axiomatic system, an artificial language, that avoided the paradoxes, that made them impossible, that outlawed them! And most mathematicians probably thought that Hilbert was right, that of course you could do this-it's just the notion that in mathematics things are absolutely clear, black or white, true or false. 
So Hilbert's idea was just an extreme, an exaggerated version of the normal notion of what mathematics is all about: the idea that we can decide and agree on the rules of the game, all of them, once and for all. The big surprise is that it turned out that this could not be done. Hilbert turned out to be wrong, but wrong in a tremendously fruitful way, because he had asked a very good question. In fact, by asking this question he actually created an entirely new field of mathematics called metamathematics.

\section{Metamathematics}

Metamathematics is mathematics turned inward, it's an introspective field of math in which you study what mathematics can achieve or can't achieve.

\section{What is Metamathematics?}

That's my field - metamathematics! In it you look at mathematics from above, and you use mathematical reasoning to discuss what mathematical reasoning can or cannot achieve. The basic idea is this: Once you entomb mathematics in an artificial language $\grave{a}$ la Hilbert, once you set up a completely formal axiomatic system, then you can forget that it has any meaning and just look at it as a game that you play with marks on paper that enables you to deduce theorems from axioms. You can forget about the meaning of this game, the game of mathematical

reasoning, it's just combinatorial play with symbols! There are certain rules, and you can study these rules and forget that they have any meaning!

What things do you look at when you study a formal axiomatic system from above, from the outside? What kind of questions do you ask?

Well, one question you can ask is if you can prove that " 0 equals $1 " ?$

$$
0=1 ?
$$

Hopefully you can't, but how can you be sure? It's hard to be sure! 
And for any question $A$, for any affirmation $A$, you can ask if it's possible to settle the matter by either proving $A$ or the opposite of $A$, not $A$.

$$
A ? \neg A ?
$$

That's called completeness.

\section{Completeness}

A formal axiomatic system is complete if you can settle any question $A$, either by proving it $(A)$, or by proving that it's false $(\neg A)$. That would be nice! Another interesting question is if you can prove an assertion $(A)$ and you can also prove the contrary assertion $(\neg A)$. That's called inconsistency, and if that happens it's very bad! Consistency is much better than inconsistency!

\section{Consistency}

So what Hilbert did was to have the remarkable idea of creating a new field of mathematics whose subject would be mathematics itself. But you can't do this until you have a completely formal axiomatic system. Because as long as any "meaning" is involved in mathematical reasoning, it's all subjective. Of course, the reason we do mathematics is because it has meaning, right? But if you want to be able to study mathematics, the power of mathematics, using mathematical methods, you have to "desiccate" it to "crystallize out" the meaning and just be left with an artificial language with completely precise rules, in fact, with one that has a mechanical proof-checking algorithm.

\section{Proof-Checking Algorithm}

The key idea that Hilbert had was to envision this perfectly desiccated or crystallized axiomatic system for all of mathematics, in which the rules would be so precise that if someone had a proof there would be a referee, there would be a mechanical procedure, which would either say "This proof obeys the rules" or "This proof is wrong; it's breaking the rules". That's how you get the criterion for mathematical truth to be completely objective and not to depend on meaning or subjective understanding: by reducing it all to calculation. Somebody says "This 
is a proof", and instead of having to submit it to a human referee who takes two years to decide if the paper is correct, instead you just give it to a machine. And the machine eventually says "This obeys the rules" or "On line 4 there's a misspelling" or "This thing on line 4 that supposedly follows from line 3, actually doesn't". And that would be the end, no appeal!

The idea was not that mathematics should actually be done this way. I think that that's calumny, that's a false accusation. I don't think that Hilbert really wanted to turn mathematicians into machines. But the idea was that if you could take mathematics and do it this way, then you could use mathematics to study the power of mathematics. And that is the important new thing that Hilbert came up with. Hilbert wanted to do this in order to reaffirm the traditional view of mathematics, in order to justify himself. . .

He proposed having one set of axioms and this formal language, this formal system, which would include all of mathematical reasoning, that we could all agree on, and that would be perfect! We'd then know all the rules of the game. And he just wanted to use metamathematics to show that this formal axiomatic system was good - that it was consistent and that it was complete - in order to convince people to accept it. This would have settled once and for all the philosophical questions "When is a proof correct?" and "What is mathematical truth?" Like this everyone could agree on whether a mathematical proof is correct or not. And in fact we used to think that this was an objective thing.

In other words, Hilbert's just saying, if it's really objective, if there's no subjective element, and a mathematical proof is either true or false, well, then there should be certain rules for deciding that and it shouldn't depend, if you fill in all the details, it shouldn't depend on interpretation. It's important to fill in all the details - that's the idea of mathematical logic, to "atomize" mathematical reasoning into such tiny steps that nothing is left to the imagination, nothing is left out! And if nothing is left out, then a proof can be checked automatically, that was Hilbert's point, that's really what symbolic logic is all about.

And Hilbert thought that he was actually going to be able to do this. He was going to formalize all of mathematics, and we were all going to agree that these were in fact the rules of the game. Then there'd be just one version of mathematical truth, not many variations. We don't 
want to have a German mathematics and a French mathematics and a Swedish mathematics and an American mathematics, no, we want a universal mathematics, one universal criterion for mathematical truth! Then a paper that is done by a mathematician in one country can be understood by a mathematician in another country. Doesn't that sound reasonable?! So you can imagine just how very, very shocking it was in 1931 when Kurt Gödel showed that it wasn't at all reasonable, that it could never be done!

1931 Kurt Gödel

\section{Kurt Gödel Discovers Incompleteness}

Gödel did this is Vienna, but he was from what I think is now called the Czech republic, from the city of Brünn or Brno. It was part of the Austro-Hungarian empire then, but now it's a separate country. And later he was at the Institute for Advanced Study in Princeton, where I visited his grave a few weeks ago. And the current owner of Gödel's house was nice enough to invite me in when he saw me examining the house [laughter] instead of calling the police! They know they're in a house that some people are interested in for historical reasons.

Okay, so what did Kurt Gödel do? Well, Gödel sort of exploded this whole view of what mathematics is all about. He came up with a famous incompleteness result, "Gödel's incompleteness theorem".

\section{Incompleteness}

And there's a lovely book explaining the way Gödel originally did it. It's by Nagel and Newman, and it's called Gödel's Proof. I read it when I was a child, and forty years later it's still in print!

What is this amazing result of Gödel's? Gödel's amazing discovery is that Hilbert was wrong, that it cannot be done, that there's no way to take all of mathematical truth and to agree on a set of rules and to have a formal axiomatic system for all of mathematics in which it is crystal clear whether something is correct or not!

More precisely, what Gödel discovered was that if you just try to deal with elementary arithmetic, with $0,1,2,3,4 \ldots$ and with addition 
and multiplication

$$
+\times 0,1,2,3,4, \ldots
$$

- this is "elementary number theory" or "arithmetic" — and you just try to have a set of axioms for this - the usual axioms are called Peano arithmetic - even this can't be done! Any set of axioms that tries to have the whole truth and nothing but the truth about addition, multiplication, and $0,1,2,3,4,5,6,7,8,9,10 \ldots$ will have to be incomplete. More precisely, it'll either be inconsistent or it'll be incomplete. So if you assume that it only tells the truth, then it won't tell the whole truth. There's no way to capture all the truth about addition, multiplication, and $0,1,2,3,4 \ldots$ ! In particular, if you assume that the axioms don't allow you to prove false theorems, then it'll be incomplete, there'll be true theorems that you cannot prove from these axioms!

This is an absolutely devastating result, and all of traditional mathematical philosophy ends up in a heap on the floor! At the time this was considered to be absolutely devastating. However you may notice that in 1931 there were also a few other problems to worry about. The situation in Europe was bad. There was a major depression, and a war was brewing. I agree, not all problems are mathematical! There's more to life than epistemology! But you begin to wonder, well, if the traditional view of mathematics isn't correct, then what is correct? Gödel's incompleteness theorem was very surprising and a terrible shock.

How did Gödel do it? Well, Gödel's proof is very clever. It almost looks crazy, it's very paradoxical. Gödel starts with the paradox of the liar, "I'm false!", which is neither true nor false.

"This statement is false!"

And what Gödel does is to construct a statement that says of itself "I'm unprovable!"

"This statement is unprovable!"

Now if you can construct such a statement in elementary number theory, in arithmetic, a mathematical statement-I don't know how you make a mathematical statement say it's unprovable, you've got to be very 
clever-but if you can do it, it's easy to see that you're in trouble. Just think about it a little bit. It's easy to see that you're in trouble. Because if it's provable, it's false, right? So you're in trouble, you're proving false results. And if it's unprovable and it says that it's unprovable, then it's true, and mathematics is incomplete. So either way, you're in trouble! Big trouble!

And Gödel's original proof is very, very clever and hard to understand. There are a lot of complicated technical details. But if you look at his original paper, it seems to me that there's a lot of LISP programming in it, or at least something that looks a lot like LISP programming. Anyway, now we'd call it LISP programming. Gödel's proof involves defining a great many functions recursively, and these are functions dealing with lists, which is precisely what LISP is all about. So even though there were no programming languages in 1931, with the benefit of hindsight you can clearly see a programming language in Gödel's original paper. And the programming language I know that's closest to it is LISP, pure LISP, LISP without side-effects, interestingly enough - that's the heart of LISP.

So this was a very, very shocking result, and people didn't really know what to make of it.

Now the next major step forward comes only five years later, in 1936, and it's by Alan Turing.

\section{Alan Turing}

\section{Alan Turing Discovers Uncomputability}

Turing's approach to all these questions is completely different from Gödel's, and much deeper. Because Turing brings it out of the closet! [Laughter] What he brings out of the closet is the computer! The computer was implicit in Gödel's paper, but this was really not visible to any ordinary mortal, not at that time, only with hindsight. And Turing really brings it out in the open.

Hilbert had said that there should be a "mechanical procedure" to decide if a proof obeys the rules or not. And Hilbert never clarified what he meant by a mechanical procedure, it was all words. But, 
Turing said, what you really mean is a machine, and a machine of a kind that we now call a Turing machine - but it wasn't called that in Turing's original paper. In fact, Turing's original paper contains a programming language, just like Gödel's paper does, what we would now call a programming language. But the two programming languages are very different. Turing's programming language isn't a high-level language like LISP, it's more like a machine language. In fact, it's a horrible machine language, one that nobody would want to use today, because it's too simple.

But Turing makes the point that even though Turing machines are very simple, even though their machine language is rather primitive, they're very flexible, very general-purpose machines. In fact, he claims, any computation that a human being can perform, should be possible to do using such a machine. Turing's train of thought now takes a very dramatic turn. What, he asks, is impossible for such a machine? What can't it do? And he immediately finds a question that no Turing machine can settle, a problem that no Turing machine can solve. That's the halting problem, the problem of deciding in advance if a Turing machine or a computer program will eventually halt.

The Halting Problem

So the shocking thing about this 1936 paper is that first of all he comes up with the notion of a general-purpose or universal computer, with a machine that's flexible, that can do what any machine can do. One calculating machine that can do any calculation, which is, we now say, a general-purpose computer. And then he immediately shows that there are limits to what such a machine can do. And how does he find something that cannot be done by any such machine? Well, it's very simple! It's the question of whether a computer program will eventually halt, with no time limit.

If you put a time limit, it's very easy. If you want to know if a program halts in a year, you just run it for a year, and either it halted or doesn't. What Turing showed is that you get in terrible trouble if there's no time limit. Now you may say, "What good is a computer program that takes more than a year, that takes more than a thousand years?! There's always a time limit!" I agree, this is pure math, this is 
not the real world. You only get in trouble with infinity! But Turing shows that if you put no time limit, then you're in real difficulties.

So this is called the halting problem. And what Turing showed is that there's no way to decide in advance if a program will eventually halt.

The Halting Problem

If it does halt, by running it you can eventually discover that, if you're just patient. The problem is you don't know when to give up. And Turing was able to show with a very simple argument which is just Cantor's diagonal argument-coming from Cantor's theory of infinite sets, by the way - I don't have time to explain all this - with a very simple argument Turing was able to show that this problem

The Halting Problem

cannot be solved.

No computer program can tell you in advance if another computer program will eventually halt or not. And the problem is the ones that don't halt, that's really the problem. The problem is knowing when to give up.

So now the interesting thing about this is that Turing immediately deduces as a corollary that if there's no way to decide in advance by a calculation if a program will halt or not, well then there cannot be any way to deduce it in advance using reasoning either. No formal axiomatic system can enable you to deduce in advance whether a program will halt or not.

Because if you can use a formal axiomatic system to always deduce whether a program will halt or not, well then, that will give you a way to calculate in advance whether a program will halt or not. You simply run through all possible deductions - you can't do this in practicebut in principle you can run through all possible proofs in size order, checking which ones are correct, until either you find a proof that the program will halt eventually or you find a proof that it's never going to halt.

This is using the idea of a completely formal axiomatic system where you don't need a mathematician-you just run through this calculation on a computer - it's mechanical to check if a proof is correct or not. So 
if there were a formal axiomatic system which always would enable you to prove, to deduce, whether a program will halt or not, that would give you a way to calculate in advance whether a program will halt or not. And that's impossible, because you get into a paradox like "This statement is false!" You get a program that halts if and only if it doesn't halt, that's basically the problem. You use an argument having the same flavor as the Russell paradox.

So Turing went more deeply into these questions than Gödel. As a student I read Gödel's proof, and I could follow it step by step: I read it in Nagel and Newman's book, which is a lovely book. It's a marvelous book, it's so understandable! It's still in print, and it was published in 1958. . . But I couldn't really feel that I was coming to grips with Gödel's proof, that I could really understand it. The whole thing seemed too delicate, it seemed too fragile, it seemed too superficial... And there's this business in the closet about computing, that's there in Gödel, but it's hidden, it's not in the open, we're not really coming to terms with it.

Now Turing is really going, I think, much deeper into this whole matter. And he's showing, by the way, that it's not just one particular axiomatic system, the one that Gödel studied, that can't work, but that no formal axiomatic system can work. But it's in a slightly different context. Gödel was really looking at $0,1,2,3,4 \ldots$ and addition and multiplication, and Turing is looking at a rather strange mathematical question, which is does a program halt or not. It's a mathematical question that did not exist at the time of Gödel's original paper. So you see, Turing worked with completely new concepts...

But Gödel's paper is not only tremendously clever, he had to have the courage to imagine that Hilbert might be wrong. There's another famous mathematician of that time, von Neumann-whose grave I found near Gödel's, by the way, at Princeton. Von Neumann was probably as clever as Gödel or anyone else, but it never occurred to him that Hilbert could be wrong. And the moment that he heard Gödel explain his result, von Neumann immediately appreciated it and immediately started deducing consequences. But von Neumann said, "I missed it, I missed the boat, I didn't get it right!" And Gödel did, so he was much more profound...

Now Turing's paper is also full of technical details, like Gödel's 
paper, because there is a programming language in Turing's paper, and Turing also gives a rather large program, which of course has bugs, because he wasn't able to run it and debug it - it's the program for a universal Turing machine. But the basic thing is the ideas, and the new ideas in Turing's work are just breathtaking! So I think that Turing went beyond Gödel, but you have to recognize that Gödel took the first step, and the first step is historically the most difficult one and takes the most courage. To imagine that Hilbert could be wrong, which never occurred to von Neumann, that was something!

\section{Discover Randomness in Pure Mathemat- ics}

Okay, so then what happened? Then World War II begins. Turing starts working on cryptography, von Neumann starts working on how to calculate atom bomb detonations, and people forget about incompleteness for a while.

This is where I show up on the scene. The generation of mathematicians who were concerned with these questions basically passes from the scene with World War II. And I'm a kid in the 1950s in the United States reading the original article by Nagel and Newman in Scientific American in 1956 that became their book.

And I didn't realize that mathematicians really preferred to forget about Gödel and go on working on their favorite problems. I'm fascinated by incompleteness and I want to understand it. Gödel's incompleteness result fascinates me, but I can't really understand it, I think there's something fishy... As for Turing's approach, I think it goes much deeper, but I'm still not satisfied, I want to understand it better.

And I get a funny idea about randomness... I was reading a lot of discussions of another famous intellectual issue when I was a kid - not the question of the foundations of mathematics, the question of the foundations of physics! These were discussions about relativity theory and cosmology and even more often about quantum mechanics, about what happens in the atom. It seems that when things are very small 
the physical world behaves in a completely crazy way that is totally unlike how objects behave here in this classroom. In fact things are random - intrinsically unpredictable - in the atom.

Einstein hated this. Einstein said that "God doesn't play dice!" By the way, Einstein and Gödel were friends at Princeton, and they didn't talk very much with anybody else, and I heard someone say that Einstein had brainwashed Gödel against quantum mechanics! [Laughter] It was the physicist John Wheeler, who told me that he once asked Gödel if there could be any connection between quantum uncertainty and Gödel's incompleteness theorem, but Gödel refused to discuss it...

Okay, so I was reading about all of this, and I began to wonder - in the back of my head I began to ask myself - could it be that there was also randomness in pure mathematics?

The idea in quantum mechanics is that randomness is fundamental, it's a basic part of the universe. In normal, everyday life we know that things are unpredictable, but in theory, in Newtonian physics and even in Einstein's relativity theory - that's all called classical as opposed to quantum physics - in theory in classical physics you can predict the future. The equations are deterministic, not probabilistic. If you know the initial conditions exactly, with infinite precision, you apply the equations and you can predict with infinite precision any future time and even in the past, because the equations work either way, in either direction. The equations don't care about the direction of time...

This is that wonderful thing sometimes referred to as Laplacian determinism. I think that it's called that because of Laplace's Essai Philosophique sur les Probabilités, a book that was published almost two centuries ago. At the beginning of this book Laplace explains that by applying Newton's laws, in principle a demon could predict the future arbitrarily far, or the past arbitrarily far, if it knew the exact conditions at the current moment. This is not the type of world where you talk about free will and moral responsibility, but if you're doing physics calculations it's a great world, because you can calculate everything!

But in the 1920s with quantum mechanics it began to look like God plays dice in the atom, because the basic equation of quantum mechanics is the Schrödinger equation, and the Schrödinger equation is an equation that talks about the probability that an electron will 
do something. The basic quantity is a probability and it's a wave equation saying how a probability wave interferes with itself. So it's a completely different kind of equation, because in Newtonian physics you can calculate the precise trajectory of a particle and know exactly how it's going to behave. But in quantum mechanics the fundamental equation is an equation dealing with probabilities! That's it, that's all there is!

You can't know exactly where an electron is and what its velocity vector is - exactly what direction and how fast it's going. It doesn't have a specific state that's known with infinite precision the way it is in classical physics. If you know very accurately where an electron is, then its velocity - its momentum - turns out to be wildly uncertain. And if you know exactly in which direction and at what speed it's going, then its position becomes infinitely uncertain. That's the infamous Heisenberg uncertainty principle, there's a trade-off, that seems to be the way the physical universe works...

It's an interesting historical fact that before people used to hate this-Einstein hated it-but now people think that they can use it! There's a crazy new field called quantum computing where the idea is to stop fighting it. If you can't lick them, join them! The idea is that maybe you can make a brand new technology using something called quantum parallelism. If a quantum computer is uncertain, maybe you can have it uncertainly do many computations at the same time! So instead of fighting it, the idea is to use it, which is a great idea.

But when I was a kid people were still arguing over this. Even though he had helped to create quantum mechanics, Einstein was still fighting it, and people were saying, "Poor guy, he's obviously past his prime!"

Okay, so I began to think that maybe there's also randomness in pure mathematics. I began to suspect that maybe that's the real reason for incompleteness. A case in point is elementary number theory, where there are some very difficult questions. Take a look at the prime numbers. Individual prime numbers behave in a very unpredictable way, if you're interested in their detailed structure. It's true that there are statistical patterns. There's a thing called the prime number theorem that predicts fairly accurately the over-all average distribution of the primes. But as for the detailed distribution of 
individual prime numbers, that looks pretty random!

So I began to think about randomness... I began to think that maybe that's what's really going on, maybe that's a deeper reason for all this incompleteness. So in the 1960s I, and independently some other people, came up with some new ideas. And I like to call this new set of ideas algorithmic information theory.

\section{Algorithmic Information Theory}

That name makes it sound very impressive, but the basic idea is just to look at the size of computer programs. You see, it's just a complexity measure, it's just a kind of computational complexity...

I think that one of the first places that I heard about the idea of computational complexity was from von Neumann. Turing came up with the idea of a computer as a mathematical concept-it's a perfect computer, one that never makes mistakes, one that has as much time and space as it needs to work - it's always finite, but the calculation can go on as long as it has to. After Turing comes up with this idea, the next logical step for a mathematician is to study the time, the work needed to do a calculation-its complexity. And in fact I think that around 1950 von Neumann suggested somewhere that there should be a new field which looks at the time complexity of computations, and that's now a very well-developed field. So of course if most people are doing that, then I'm going to try something else!

My idea was not to look at the time, even though from a practical point of view time is very important. My idea was to look at the size of computer programs, at the amount of information that you have to give a computer to get it to perform a given task. From a practical point of view, the amount of information required isn't as interesting as the running time, because of course it's very important for computers to do things as fast as possible... But it turns out that from a conceptual point of view, it's not that way at all. I believe that from a fundamental philosophical point of view, the right question is to look at the size of computer programs, not at the time. Why? - Besides the fact that it's my idea so obviously I'm going to be prejudiced! The reason is because program-size complexity connects with a lot of fundamental stuff in physics. 
You see, in physics there's a notion called entropy, which is how disordered a system is. Entropy played a particularly crucial role in the work of the famous 19th century physicist Boltzmann,

\section{Ludwig Boltzmann}

and it comes up in the field of statistical mechanics and in thermodynamics. Entropy measures how disordered, how chaotic, a physical system is. A crystal has low entropy, and a gas at high temperature has high entropy. It's the amount of chaos or disorder, and it's a notion of randomness that physicists like.

And entropy is connected with some fundamental philosophical questions - it's connected with the question of the arrow of time, which is another famous controversy. When Boltzmann invented this wonderful thing called statistical mechanics - his theory is now considered to be one of the masterpieces of 19th century physics, and all physics is now statistical physics - he ended up by committing suicide, because people said that his theory was obviously wrong! Why was it obviously wrong? Because in Boltzmann's theory entropy has got to increase and so there's an arrow of time. But if you look at the equations of Newtonian physics, they're time reversible. There's no difference between predicting the future and predicting the past. If you know at one instant exactly how everything is, you can go in either direction, the equations don't care, there's no direction of time, backward is the same as forward.

But in everyday life and in Boltzmann statistical mechanics, there is a difference between going backward and forward. Glasses break, but they don't reassemble spontaneously! And in Boltzmann's theory entropy has got to increase, the system has to get more and more disordered. But people said, "You can't deduce that from Newtonian physics!" Boltzmann was pretending to. He was looking at a gas. The atoms of a gas bounce around like billiard balls, it's a billiard ball model of how a gas works. And each interaction is reversible. If you run the movie backwards, it looks the same. If you look at a small portion of a gas for a small amount of time, you can't tell whether you're seeing the movie in the right direction or the wrong direction.

But Boltzmann gas theory says that there is an arrow of time - a system will start off in an ordered state and will end up in a very mixed 
up disordered state. There's even a scary expression in German, heat death. People said that according to Boltzmann's theory the universe is going to end up in a horrible ugly state of maximum entropy or heat death! This was the dire prediction! So there was a lot of controversy about his theory, and maybe that was one of the reasons that Boltzmann killed himself.

And there is a connection between my ideas and Boltzmann's, because looking at the size of computer programs is very similar to this notion of the degree of disorder of a physical system. A gas takes a large program to say where all its atoms are, but a crystal doesn't take as big a program, because of its regular structure. Entropy and program-size complexity are closely related...

This idea of program-size complexity is also connected with the philosophy of the scientific method. You've heard of Occam's razor, of the idea that the simplest theory is best? Well, what's a theory? It's a computer program for predicting observations. And the idea that the simplest theory is best translates into saying that a concise computer program is the best theory. What if there is no concise theory, what if the most concise program or the best theory for reproducing a given set of experimental data is the same size as the data? Then the theory is no good, it's cooked up, and the data is incomprehensible, it's random. In that case the theory isn't doing a useful job. A theory is good to the extent that it compresses the data into a much smaller set of theoretical assumptions. The greater the compression, the better!That's the idea...

So this idea of program size has a lot of philosophical resonances, and you can define randomness or maximum entropy as something that cannot be compressed at all. It's an object with the property that basically the only way you can describe it to someone is to say "this is it" and show it to them. Because it has no structure or pattern, there is no concise description, and the thing has to be understood as "a thing in itself", it's irreducible.

$$
\text { Randomness }=\text { Incompressibility }
$$

The other extreme is an object that has a very regular pattern so you can just say that it's "a million 0s" or "half a million repetitions 
of $01 "$, pairs 01, 01, 01 repeated half a million times. These are very long objects with a very concise description. Another long object with a concise description is an ephemeris, I think it's called that, it's a table giving the positions of the planets as seen in sky, daily, for a year. You can compress all this astronomical information into a small FORTRAN program that uses Newtonian physics to calculate where the planets will be seen in the sky every night.

But if you look at how a roulette wheel behaves, then there is no pattern, the series of outcomes cannot be compressed. Because if there were a pattern, then people could use it to win, and having a casino wouldn't be such a good business! The fact that casinos make lots of money shows that there is no way to predict what a roulette wheel will do, there is no pattern - the casinos make it their job to ensure that!

So I had this new idea, which was to use program-size complexity to define randomness. And when you start looking at the size of computer programs - when you begin to think about this notion of program-size or information complexity instead of run-time complexity-then the interesting thing that happens is that everywhere you turn you immediately find incompleteness! You immediately find things that escape the power of mathematical reasoning, things that escape the power of any computer program. It turns out that they're everywhere!

It's very dramatic! In only three steps we went from Gödel, where it's very surprising that there are limits to reasoning, to Turing, where it looks much more natural, and then when you start looking at program size, well, incompleteness, the limits of mathematics, it just hits you in the face! Why?! Well, the very first question that you ask in my theory gets you into trouble. What's that? Well, in my theory I measure the complexity of something by the size of the smallest computer program for calculating it. But how can I be sure that I have the smallest computer program?

Let's say that I have a particular calculation, a particular output, that I'm interested in, and that I have this nice, small computer program that calculates it, and I think that it's the smallest possible program, the most concise one that produces this output. Maybe a few friends of mine and I were trying to do it, and this was the best program that we came up with; nobody did any better. But how can you be sure? Well, the answer is that you can't be sure. It turns out you 
can never be sure! You can never be sure that a computer program is what I like to call elegant, namely that it's the most concise one that produces the output that it produces. Never ever! This escapes the power of mathematical reasoning, amazingly enough.

But for any computational task, once you fix the computer programming language, once you decide on the computer programming language, and if you have in mind a particular output, there's got to be at least one program that is the smallest possible. There may be a tie, there may be several, right?, but there's got to be at least one that's smaller than all the others. But you can never be sure that you've found it!

And the precise result, which is one of my favorite incompleteness results, is that if you have $N$ bits of axioms, you can never prove that a program is elegant - smallest possible - if the program is more than $N$ bits long. That's basically how it works. So any given set of mathematical axioms, any formal axiomatic system in Hilbert's style, can only prove that finitely many programs are elegant, are the most concise possible for their output.

To be more precise, you get into trouble with an elegant program if it's larger than a computerized version of the axioms - It's really the size of the proof-checking program for your axioms. In fact, it's the size of the program that runs through all possible proofs producing all possible theorems. If you have in mind a particular programming language, and you need a program of a certain size to implement a formal axiomatic system, that is to say, to write the proof-checking algorithm and to write the program that runs through all possible proofs filtering out all the theorems, if that program is a certain size in a language, and if you look at programs in that same language that are larger, then you can never be sure that such a program is elegant, you can never prove that such a program is elegant using the axioms that are implemented in the same language by a smaller program. That's basically how it works.

So there are an infinity of elegant programs out there. For any computational task there's got to be at least one elegant program, and there may be several, but you can never be sure except in a finite number of cases. That's my result, and I'm very proud of it!-Another can of soda? Thanks a lot! My talk would be much more interesting if this were wine or beer! [Laughter] 
So it turns out that you can't calculate the program-size complexity, you can never be sure what the program-size complexity of anything is. Because to determine the program-size complexity of something is to know the size of the most concise program that calculates it - but that means - it's essentially the same problem - then I would know that this program is the most concise possible, I would know that it's an elegant program, and you can't do that if the program is larger than the axioms. So if it's $N$ bits of axioms, you can never determine the program-size complexity of anything that has more than $N$ bits of complexity, which means almost everything, because almost everything has more than $N$ bits of complexity. Almost everything has more complexity than the axioms that you're using.

Why do I say that? The reason for using axioms is because they're simple and believable. So the sets of axioms that mathematicians normally use are fairly concise, otherwise no one would believe in them! Which means that in practice there's this vast world of mathematical truth out there, which is an infinite amount of information, but any given set of axioms only captures a tiny finite amount of this information! And that's why we're in trouble, that's my bottom line, that's my final conclusion, that's the real dilemma.

So in summary, I have two ways to explain why I think Gödel incompleteness is natural and inevitable rather than mysterious and surprising. The two ways are - that the idea of randomness in physics, that some things make no sense, also happens in pure mathematics, is one way to say it. But a better way to say it, is that mathematical truth is an infinite amount of information, but any particular set of axioms just has a finite amount of information, because there are only going to be a finite number of principles that you've agreed on as the rules of the game. And whenever any statement, any mathematical assertion, involves more information than the amount in those axioms, then it's very natural that it will escape the ability of those axioms.

So you see, the way that mathematics progresses is you trivialize everything! The way it progresses is that you take a result that originally required an immense effort, and you reduce it to a trivial corollary of a more general theory!

Let me give an example involving Fermat's "last theorem", namely 
the assertion that

$$
x^{n}+y^{n}=z^{n}
$$

has no solutions in positive integers $x, y, z$, and $n$ with $n$ greater than 2. Andrew Wiles's recent proof of this is hundreds of pages long, but, probably, a century or two from now there will be a one-page proof! But that one-page proof will require a whole book inventing a theory with concepts that are the natural concepts for thinking about Fermat's last theorem. And when you work with those concepts it'll appear immediately obvious - Wiles's proof will be a trivial afterthought - because you'll have imbedded it in the appropriate theoretical context.

And the same thing is happening with incompleteness.

Gödel's result, like any very fundamental basic result, starts off by being very mysterious and complicated, with a long impenetrable proof. People said about Gödel's original paper the same thing that they said about Einstein's theory of relativity, which is that there are less than five people on this entire planet who understand it. The joke was that Eddington, astronomer royal Sir Arthur Eddington, is at a formal dinner party - this was just after World War I - and he's introduced as one of the three men who understands Einstein's theory. And he says, "Let's see, there's Einstein, and there's me, but who's the other guy?" I'm ruining this joke! [Laughter]

So in 1931 Gödel's proof was like that. If you look at his original paper, it's very complicated. The details are programming details we would say now - really it's a kind of complication that we all know how to handle now - but at the time it looked very mysterious. This was a 1931 mathematics paper, and all of a sudden you're doing what amounts to LISP programming, thirty years before LISP was invented! And there weren't even any computers then!

But when you get to Turing, he makes Gödel's result seem much more natural. And I think that my idea of program-size complexity and information - really, algorithmic information content-makes Gödel's result seem more than natural, it makes it seem, I'd say, obvious, inevitable. But of course that's the way it works, that's how we progress. 


\section{Where Do We Go from Here?!}

I should say, though, that if this were really true, if it were that simple, then that would be the end of the field of metamathematics. It would be a sad thing, because it would mean that this whole subject is dead. But I don't think that it is!

You know, I've been giving versions of this talk for many years. I make a career, a profession out of it! It's tourism, it's the way I get to see the world! It's a nice way to travel!... In these talks I like to give examples of things that might escape the power of normal mathematical reasoning. And my favorite examples were Fermat's last theorem, the Riemann hypothesis, and the four-color conjecture. When I was a kid these were the three most outstanding open questions in all of mathematics.

But a funny thing happened. First the four-color conjecture was settled by a computer proof, and recently the proof has been greatly improved. The latest version has more ideas and less computation, so that's a big step forward. And then Wiles settled Fermat's last theorem. There was a misstep, but now everyone's convinced that the new proof is correct.

In fact, I was at a meeting in June 1993, when Wiles was presenting his proof in Cambridge. I wasn't there, but I was at a meeting in France, and the word was going around by e-mail that Wiles had done it. It just so happened that I was session chairman, and at one point the organizer of the whole meeting said, "Well, there's this rumor going around, why don't we make an announcement. You're the session chairman, you do it!" So I got up and said, "As some of you may have heard, Andrew Wiles has just demonstrated Fermat's last theorem." And there was silence! But afterwards two people came up and said, "You were joking, weren't you?" [Laughter] And I said, "No, I wasn't joking." It wasn't April 1st!

Fortunately the Riemann hypothesis is still open at this point, as far as I know!

But I was using Fermat's last theorem as a possible example of incompleteness, as an example of something that might be beyond the power of the normal mathematical methods. I needed a good example, because people used to say to me, "Well, this is all very well and good, 
AIT is a nice theory, but give me an example of a specific mathematical result that you think escapes the power of the usual axioms." And I would say, well, maybe Fermat's last theorem!

So there's a problem. Algorithmic information theory is very nice and shows that there are lots of things that you can't prove, but what about individual mathematical questions? How about a natural mathematical question? Can these methods be applied? Well, the answer is no, my methods are not as general as they sound. There are technical limitations. I can't analyze Fermat's last theorem with these methods. Fortunately! Because if I had announced that my methods show that Fermat's last theorem can't be settled, then it's very embarrassing when someone settles it!

So now the question is, how come in spite of these negative results, mathematicians are making so much progress? How come mathematics works so well in spite of incompleteness? You know, I'm not a pessimist, but my results have the wrong kind of feeling about them, they're much too pessimistic!

So I think that a very interesting question now is to look for positive results... There are already too many negative results! If you take them at face value, it would seem that there's no way to do mathematics, that mathematics is impossible. Fortunately for those of us who do mathematics, that doesn't seem to be the case. So I think that now we should look for positive results... The fundamental questions, like the questions of philosophy, they're great, because you never exhaust them. Every generation takes a few steps forward... So I think there's a lot more interesting work to be done in this area.

And here's another very interesting question: Program size is a complexity measure, and we know that it works great in metamathematics, but does it have anything to do with complexity in the real world? For example, what about the complexity of biological organisms? What about a theory of evolution?

Von Neumann talked about a general theory of the evolution of life. He said that the first step was to define complexity. Well, here's a definition of complexity, but it doesn't seem to be the correct one to use in theoretical biology. And there is no such thing as theoretical biology, not yet!

As a mathematician, I would love it if somebody would prove a 
general result saying that under very general circumstances life has to evolve. But I don't know how you define life in a general mathematical setting. We know it when we see it, right? If you crash into something alive with your car, you know it! But as a mathematician I don't know how to tell the difference between a beautiful deer running across the road and the pile of garbage that my neighbor left out in the street! Well, actually that garbage is connected with life, it's the debris produced by life...

So let's compare a deer with a rock instead. Well, the rock is harder, but that doesn't seem to go to the essential difference that the deer is alive and the rock is a pretty passive object. It's certainly very easy for us to tell the difference in practice, but what is the fundamental difference? Can one grasp that mathematically?

So what von Neumann was asking for was a general mathematical theory. Von Neumann used to like to invent new mathematical theories. He'd invent one before breakfast every day: the theory of games, the theory of self-reproducing automata, the Hilbert space formulation of quantum mechanics... Von Neumann wrote a book on quantum mechanics using Hilbert spaces - that was done by von Neumann, who had studied under Hilbert, and who said that this was the right mathematical framework for doing quantum mechanics.

Von Neumann was always inventing new fields of mathematics, and since he was a childhood hero of mine, and since he talked about Gödel and Turing, well, I said to myself, if von Neumann could do it, I think I'll give it a try. Von Neumann even suggested that there should be a theory of the complexity of computations. He never took any steps in that direction, but I think that you can find someplace where he said that this has got to be an interesting new area to develop, and he was certainly right.

Von Neumann also said that we ought to have a general mathematical theory of the evolution of life... But we want it to be a very general theory, we don't want to get involved in low-level questions like biochemistry or geology... He insisted that we should do things in a more general way, because von Neumann believed, and I guess I do too, that if Darwin is right, then it's probably a very general thing.

For example, there is the idea of genetic programming, that's a computer version of this. Instead of writing a program to do something, 
you sort of evolve it by trial and error. And it seems to work remarkably well, but can you prove that this has got to be the case? Or take a look at Tom Ray's Tierra... Some of these computer models of biology almost seem to work too well - the problem is that there's no theoretical understanding why they work so well. If you run Ray's model on the computer you get these parasites and hyperparasites, you get a whole ecology. That's just terrific, but as a pure mathematician I'm looking for theoretical understanding, I'm looking for a general theory that starts by defining what an organism is and how you measure its complexity, and that proves that organisms have to evolve and increase in complexity. That's what I want, wouldn't that be nice?

And if you could do that, it might shed some light on how general the phenomenon of evolution is, and whether there's likely to be life elsewhere in the universe. Of course, even if mathematicians never come up with such a theory, we'll probably find out by visiting other places and seeing if there's life there... But anyway, von Neumann had proposed this as an interesting question, and at one point in my deluded youth I thought that maybe program-size complexity had something to do with evolution... But I don't think so anymore, because I was never able to get anywhere with this idea...

So I think that there's a lot of interesting work to be done! And I think that we live in exciting times. In fact, sometimes I think that maybe they're even a little bit too exciting!... And I hope that if this talk were being given a century from now, in 2099, there would be another century of exciting controversy about the foundations of mathematics to summarize, one with different concerns and preoccupations... It would be interesting to hear what that talk would be like a hundred years from now! Maybe some of you will be there! Or give the talk even! Thank you very much! [Laughter \& Applause]

\section{Further Reading}

1. G. J. Chaitin, The Unknowable, Springer-Verlag, 1999.

2. G. J. Chaitin, The Limits of Mathematics, Springer-Verlag, 1998. 